\title{
Near-IR bispectrum speckle interferometry, AO imaging polarimetry, and radiative transfer modeling of the proto-planetary nebula Frosty Leonis
}

\author{
K. Murakawa ${ }^{1}$, K. Ohnaka ${ }^{1}$, T. Driebe ${ }^{1}$, K.-H. Hofmann ${ }^{1}$, S. Oya ${ }^{2}$, D. Schertl ${ }^{1}$, and G. Weigelt ${ }^{1}$ \\ ${ }^{1}$ Max-Planck-Institut für Radioastronomie, Auf dem Hügel 69, 53121 Bonn, Germany \\ e-mail: murakawa@mpifr-bonn.mpg.de \\ 2 Subaru Telescope, 650 North A'ohoku place, Hilo, HI 96720, USA
}

Received 6 September 2007 / Accepted 16 July 2008

ABSTRACT

\begin{abstract}
Aims. We combined bispectrum speckle interferometry, adaptive optics (AO) imaging polarimetry, and radiative transfer modeling of polarized light to derive various physical properties of the proto-planetary nebula Frosty Leo.

Methods. We performed bispectrum $K^{\prime}$-band speckle interferometry and $H$ - and $K$-band imaging polarimetry of Frosty Leo using the ESO $3.6 \mathrm{~m}$ telescope and the AO-equipped CIAO instrument of the $8 \mathrm{~m}$ Subaru telescope, respectively. Two-dimensional radiative transfer modeling was carried out in order to obtain a quantitative interpretation of our observations.

Results. Our diffraction-limited speckle image shows distinct hourglass-shaped, point-symmetric bipolar lobes, an equatorial dust lane, and complex clumpy structures in the lobes. Our polarimetric data display a centro-symmetric polarization vector pattern with $P \sim 30-50 \%$ in the bipolar lobes and a polarization disk between them. The polarization images also reveal an elongated region with low polarization along a position angle of $-45^{\circ}$. The observations suggest that this region has a low dust density and was carved out by a jet-like outflow. Our radiative transfer modeling can simultaneously explain the observed spectral energy distribution, the intensity distribution of the hourglass-shaped lobes, and our polarization images if we use two grain species with sizes of $0.005 \leq a \leq 2.0 \mu \mathrm{m}$ at latitudes between $-2^{\circ}$ and $+2^{\circ}$, and $0.005 \leq a \leq 0.7 \mu \mathrm{m}$ in the bipolar lobes. Assuming a distance of $3 \mathrm{kpc}$, an expansion velocity of $25 \mathrm{~km} \mathrm{~s}^{-1}$, and a gas-to-dust mass ratio of 160 , we derive a dust mass of the disk of $2.85 \times 10^{-3} M_{\odot}$, a gas mass-loss rate of $8.97 \times 10^{-3} M_{\odot} \mathrm{yr}^{-1}$, and a total envelope mass of $4.23 M_{\odot}$.
\end{abstract}

Key words. Stars: AGB and post-AGB - circumstellar matter - radiative transfer - polarization - method: numerical methods: observational

\section{Introduction}

Frosty Leo, also known as IRAS $09371+1212$, is an oxygenrich proto-planetary nebula (PPN). The distinct bipolar appearance of the nebula is remarkable. Previous optical and nearinfrared (NIR) imaging resolved the hourglass-shaped lobes (e.g. Rouan et al. 1988; Beuzit et al. 1994; Roddier et al. 1995; Lopez et al. 2001). Dougados et al. (1990) and Scarrott \& Scarrott (1994) performed imaging polarimetry in the $J K$ and $B V R I$ bands, respectively, and their results revealed centrosymmetrically aligned polarization vectors in the bipolar lobes and a polarization disk between them. These polarization signatures are convincing evidence that a) in the optical and NIR, the nebula is seen in light scattered by dust and b) that a thick equatorial dust concentration is present. More complex structures such as ansae and jets have also been detected in the optical and NIR on $\sim 10^{\prime \prime}$ scales (Morris \& Reipurth 1990; Langill et al. 1994; Sahai et al. 2000). Because these morphologies are thought to characterize the activities of the star and the circumstellar environment at late evolutionary phases, Frosty Leo is a key target which can provide important insights to answer open questions on evolved stars; namely, how bipolar PNs form in the early post-AGB phase and what physical conditions exist in the nebula.

In this paper, we present NIR diffraction-limited bispectrum speckle interferometry and imaging polarimetry of the Frosty Leo nebula. The observational details and results are discussed in Sect.2. Our radiative transfer calculations are described in Sect. 3 .

\section{Observations and results}

\subsection{K'-band bispectrum speckle interferometry}

We carried out $K^{\prime}$-band bispectrum speckle interferometry of Frosty Leo using the ESO $3.6 \mathrm{~m}$ telescope at La Silla on January 29,2007 . The central wavelength and the bandwidth of the $K^{\prime}$-band filter used were $\lambda_{\mathrm{c}}=2.161 \mu \mathrm{m}$ and $\Delta \lambda=0.34 \mu \mathrm{m}$, respectively. The pixel scale of our HAWAII camera was $46.68 \mathrm{mas} \mathrm{pix}^{-1}$. The natural $K^{\prime}$-band seeing was $00^{\prime} 9$. We obtained 1100 speckle interferograms of the target and 1100 of the unresolved star TYC 824-1188-1 $\left(m_{K}=7.4\right)$. The exposure time per frame was $600 \mathrm{~ms}$. The unresolved star was used for the compensation of the atmospheric speckle transfer function.

A diffraction-limited image of Frosty Leo with 0 ' 12 resolution was reconstructed from all speckle interferograms using the bispectrum speckle interferometry method (Weigelt 1977; Lohmann et al. 1983; Hofmann \& Weigelt 1986). The modulus of the object Fourier transform (visibility function) was determined with the speckle interferometry method (Labeyrie 1970).

Figure 1 presents the reconstructed $K^{\prime}$-band speckle image. The field of view (FOV) is 7". $4 \times 77^{\prime \prime} 4$. The image shows the well-known hourglass-like bipolar lobes extending north and south, and a distinct dust lane (narrow waist) along the position angle (PA) $75^{\circ}$. The resolution and the signal-to-noise ratio (SNR) of the reconstructed image are high enough to resolve the 


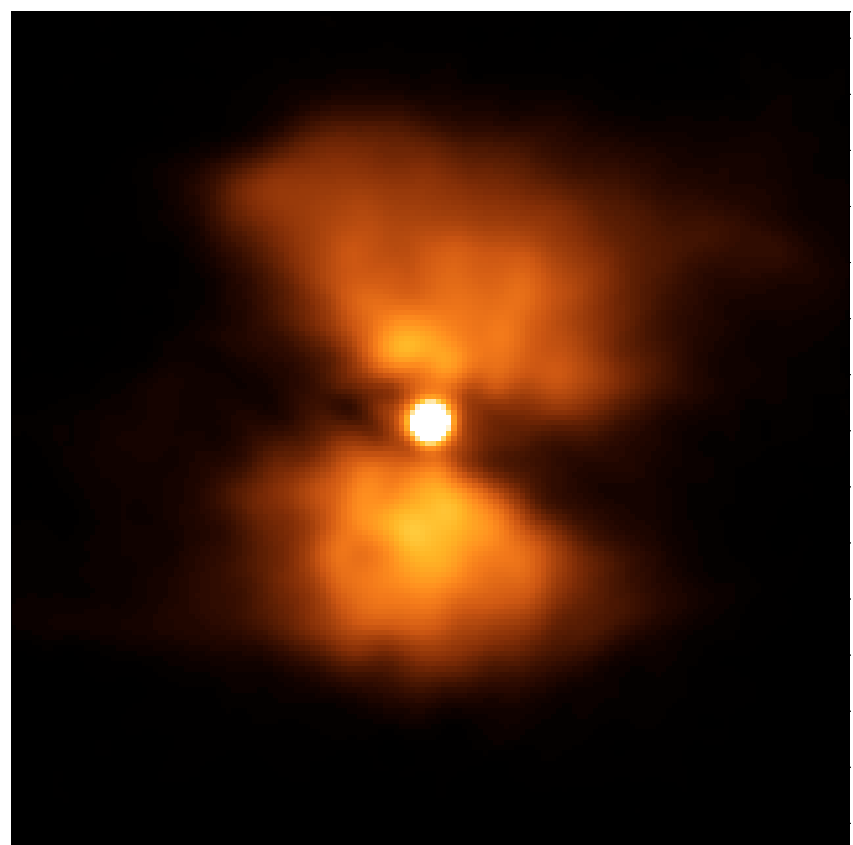

Fig. 1. Diffraction-limited ( $0{ }^{\prime} 12$ resolution) $K^{\prime}$-band image of Frosty Leo reconstructed with the bispectrum speckle interferometry method. The field of view is $77^{\prime \prime} 4 \times 7^{\prime \prime}$. 4 . North is up and east is to the left. The image shows the well-known hourglass-shaped bipolar nebula, the clumpy intensity distribution resolved in both lobes, a dust lane between the lobes, and the central star.

clumpy structure of the two lobes. The central object looks like a single star. We find no evidence of a double star with a separation of $0 . ' 19$, as reported by Roddier et al. (1995).

\subsection{HK-band AO imaging polarimetry}

We obtained polarimetric images using the Coronagraphic Imager with Adaptive Optics (CIAO) with the 36-element adaptive optics (AO) on the $8 \mathrm{~m}$ Subaru telescope in the $H$ and $K$ bands on January 10, 2004. The central wavelengths and the bandwidths of the $H$ - and $K$-band filters are $\lambda_{H}=1.65 \mu \mathrm{m}$ and $\Delta \lambda_{H}=0.30 \mu \mathrm{m}$, and $\lambda_{K}=2.20 \mu \mathrm{m}$ and $\Delta \lambda_{K}=0.34 \mu \mathrm{m}$, respectively. We used the medium resolution camera with a pixel scale of 0.0217 arcsec pix $^{-1}$. The seeing was $\sim 0.7$ in the $R$ band. Since there is no bright single star near Frosty Leo which would be appropriate for AO wavefront sensing $\left(m_{R}\right.$ brighter than 16 mag and a separation less than $20^{\prime \prime}$ ) and the target is sufficiently bright at $\lambda \sim 0.8 \mu \mathrm{m}$ for wavefront sensing $\left(m_{R}=9.7\right)$, on-source AO wavefront sensing was applied. We followed the observation sequence described in a previous paper (Murakawa et al. 2005). In order to measure the linear polarization, we obtained four sets of images with half-wave plate (HWP) orientations of $0^{\circ}, 45^{\circ}, 22.5^{\circ}$, and $67.5^{\circ}$. The exposure time per frame was $10 \mathrm{~s}$. Three frames were obtained for each orientation of the HWP, and five dither offsets of $8^{\prime \prime}$ separation were performed. The total integration time was $600 \mathrm{~s}$. We also observed HD 38563C $\left(m_{H}=9.12, P_{H}=3.68 \pm 0.10 \%, m_{K}=8.76\right.$ and $\left.P_{K}=2.21 \pm 0.55 \%\right)$ from a polarimetric standard catalogue (Whittet et al. 1992).

We reduced the recorded data as described in our previous papers (Murakawa et al. 2004, 2005). After preprocessing (subtraction of the dark frame and flat fielding), we obtained images of the Stokes $I Q U$ parameters, the degree of polarization $(P)$, the polarization position angle $(\theta)$, as well as their error images. The signal-to-noise ratios of the Stokes $I$ images per pixel are 50-300, and the errors of linear polarization per pixel are $0.3-2 \%$. The typical accuracy of the polarization position angle per pixel is $0.5^{\circ}-2.0^{\circ}$ in the (NS) bipolar lobes (with $P \sim 30 \%$ ) and $\pm 20^{\circ}$ between them (with $P \sim 1 \%$ ).

Figures $2 \mathrm{a}$ and $\mathrm{b}$ show the total intensity (Stokes $I$ ) images in the $H$ and $K$ bands. The FOV is $10^{\prime \prime} \times 10^{\prime \prime}$. The overall appearance is in good agreement with our speckle image. Figure 3 shows a cartoon of the observational results. In our AO images, bipolar lobes are detected up to $4-5^{\prime \prime}$ from the central star, and ridge-like features are seen towards the northwest, northeast, southeast, and southwest. These are approximately at the same PAs as the jets, indicated by $\mathrm{J}^{\prime}{ }^{\prime}, \mathrm{J} 2^{\prime}, \mathrm{J} 1$, and $\mathrm{J} 2$, respectively, in the HST optical image (Sahai et al. 2000). In contrast to our speckle image in Fig. 1, the point-spread functions of our AO images have elliptical shapes with dimensions of 0 ' $18 \times 0.28$ in the $H$ band and $0.16 \times 0.28$ in the $K$ band, respectively. The elongated PSFs are caused by AO wavefront-correction errors. Such an imperfect correction can occur if the object used for wavefront sensing is an extended asymmetric nebula such as Frosty Leo.

Figures $2 \mathrm{c}$ and d show the observed $H$ - and $K$-band polarization vectors (i.e., degree of polarization and position angle of the electric field) and the degree of polarization (pseudo color image). Contour lines of the Stokes $I$ images are also plotted for comparison. We see a centro-symmetric polarization vector pattern in the bipolar lobes and a polarization disk extending $\sim 5^{\prime \prime}$ along the dust lane. The degree of polarization in the lobes reaches up to $35-50 \%$ and $40-55 \%$ in the $H$ and $K$ bands, respectively. These results are in good agreement with those previously reported by Dougados et al. (1990) and Scarrott \& Scarrott (1994). Furthermore, we discovered two new polarization signatures. The first one is an elongated region with low polarizations $(P \sim 20 \%)$ lying at a PA of $-45^{\circ}$, which is roughly the direction towards the northwest ridge. The low polarization can be explained by the dependence of polarization on the grain size and scattering angle (e.g. Fig. 2, Fischer et al. 1994). Since larger grains produce a lower polarization than smaller grains, one possibility to explain this feature is a tube-like structure filled with large grains. The other possibility is also a tube-like structure but with low densities lying near the plane of sky. The degree of polarization peaks near a scattering angle of $90^{\circ}$. If the dust density is low near the plane of sky in the line of sight, a large fraction of light from this direction is dominated by forward or backward scattered light, which has low polarizations. Taking into account that the PAs of the jet-like structure (indicated as $\mathrm{J}^{\prime}$ ) and the $\mathrm{CO}$ outflow (Castro-Carrizo et al. 2005) are consistent with those of the elongated region with low polarization (see Fig. 3), this region is more likely to be a tube-like structure with low densities, which was probably carved out by the jet, than one filled with large grains. The second newly discovered signature is the horizontal alignment of polarization vectors in a region between the polarization null points within the dust lane. The direction of alignment is tilted against the polarization disk. In this region, the linear polarization is $P_{H} \sim 12.2 \%$ and $P_{K} \sim 12.8 \%$ at a 0.2 aperture, which is much higher than the estimated error of $<2 \%$.

\section{Radiation transfer calculations}

\subsection{Monte Carlo code}

We performed radiative transfer modeling to investigate the physical properties of Frosty Leo's nebula. We used our Monte Carlo radiative transfer code mcsim_mpi (Ohnaka et al. 2006). It simulates scattering, absorption, and reemission by spherical dust grains in an arbitrary three-dimensional model 

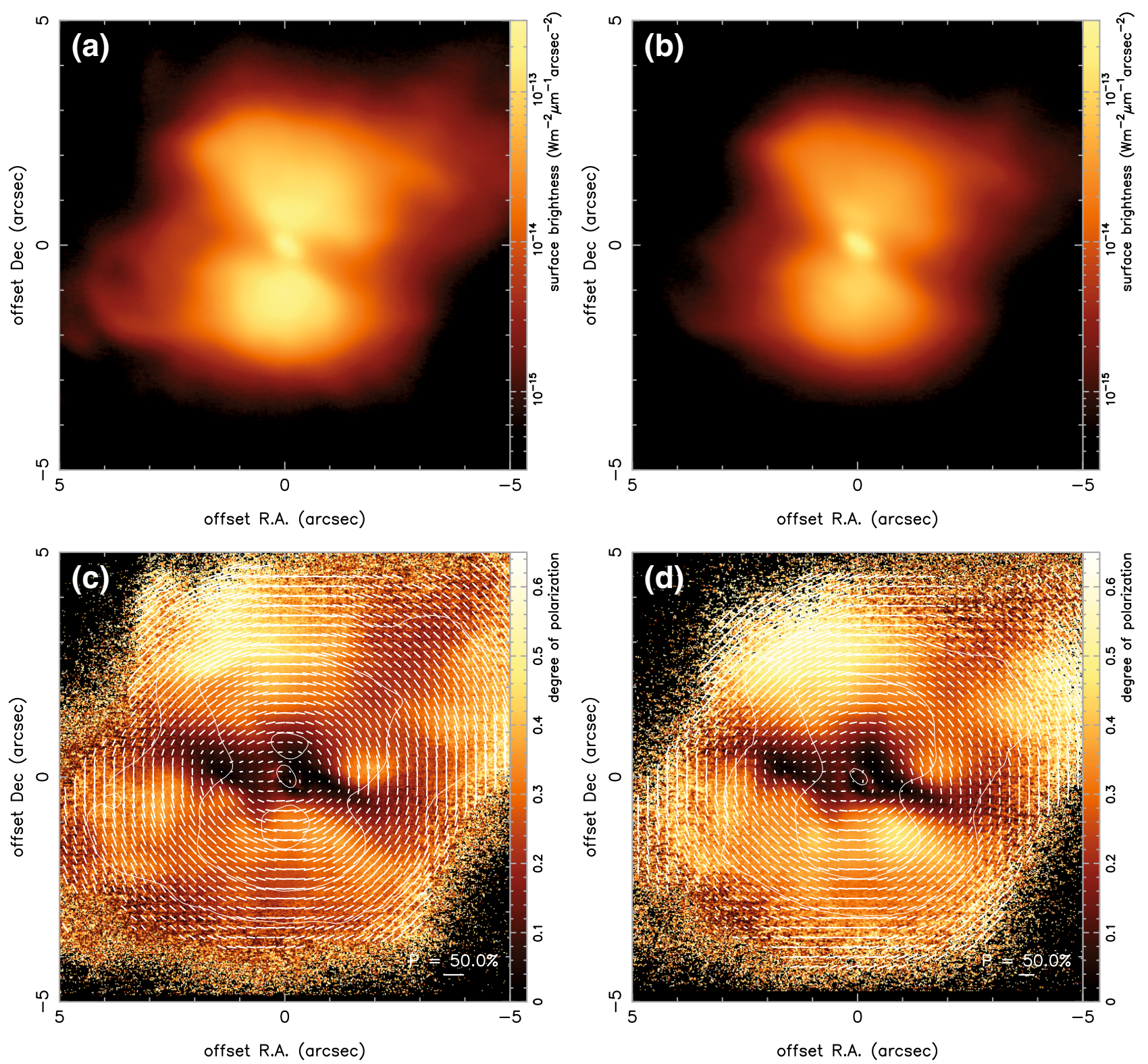

Fig. 2. AO imaging polarimetry of Frosty Leo obtained with the Subaru telescope, a) and b) are the total intensity (Stokes $I$ ) images in the $H$ and $K$ bands, respectively; c) and d) show the observed $H$ - and $K$-band polarization vectors (i.e., degree of polarization and position angle of the electric field) and the degree of polarization (gray-scale heat image). The polarization vector lines are drawn every 10 pixels. Contour plots with 2.5 mag spacing of the total intensity images are overlaid for comparison.

geometry, computes SEDs and dust temperatures in radiation equilibrium, and generates Stokes $I Q U V$ images. This code has a "full interaction mode" and a "fixed wavelength mode" (or scattering mode). In the former mode, photon packets are emitted at all wavelengths according to the spectrum of the central star, and absorption, reemission, and scattering are simulated every time a photon packet interacts with dust grains. However, in this mode it is very inefficient to obtain the output Stokes images, and the resulting images suffer from large Monte Carlo noise. If thermal emission from dust is negligible at a specific wavelength, the fixed wavelength mode is preferred. This mode treats only monochromatic light at a given wavelength. Photon packets are always scattered at interacting points, and the photon weight is reduced by the dust albedo $\omega$ (see also the paper by Witt 1977). Therefore, the fixed wavelength mode generates the output Stokes images more efficiently than the full interaction mode.
We assume dust grains with sizes between $a_{\min }$ and $a_{\max }$, a power-law size distribution (Mathis et al. 1977), and with or without a mantle with a volume fraction of the mantle $f_{\mathrm{m}}$. Opacities and scattering matrix components are computed according to Mie's scattering theory (e.g. Bohren \& Huffmann 1983) and their size-averaged values are used (see also Sect. 3.1 in Murakawa et al. 2007). We implement the algorithm proposed by Fischer et al. (1994), which determines the direction of scattered light depending on the incident polarization status. Such a treatment of scattered light, as well as the possibility of multiple scattering, allows a proper analysis of the radiation of polarized light. Several advantages of these techniques have been reported (e.g. Bastien \& Ménard 1988; Fischer et al. 1994). Before applying the modified code to Frosty Leo, we carried out a series of tests, as described in the appendix. 


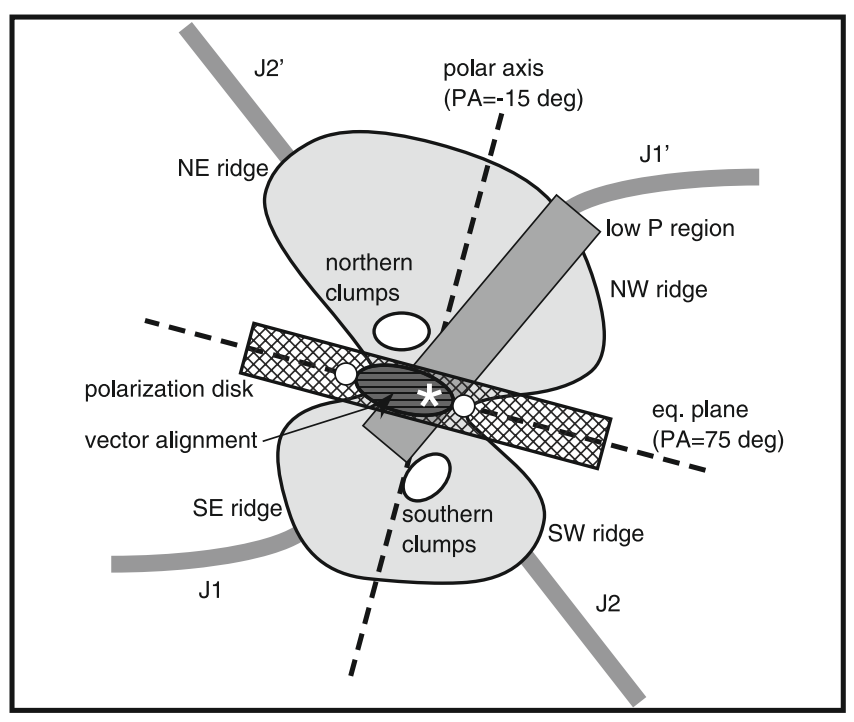

Fig. 3. A cartoon of Frosty Leo illustrating the features detected in our speckle and polarization images. The location of the central star and the polarization null points are indicated with a white asterisk in the middle of the nebula and open circles on both sides of the region with polarization alignment, respectively. The directions of four jet-like features, indicated by $\mathrm{J} 1, \mathrm{~J}^{\prime}, \mathrm{J} 2$, and $\mathrm{J} 2^{\prime}$, which were detected in an HST optical image (Sahai et al. 2000), are also drawn for comparison.

\subsection{Numerical simulations}

We started our 2-D modeling of Frosty Leo with single grain species models, which are similar to previously reported ones, and compare them with the observational results of SEDs, total intensity images, and polarization images. As described in Sect. 3.2.1, previous estimations of the grain size depended on the modeling methods. Thus, we also attempted to optimize the grain parameters in our simulations by studying the effects of different grain parameters on the SED, total intensity images, and polarization images. As will be described in the following sections, we found some difficulties in explaining our observations with single grain species models. Therefore, in subsequent simulations, we tried to construct more plausible models by introducing two different grain species.

\subsubsection{Model assumptions}

Although Frosty Leo is a well-studied PPN, there are still several uncertainties concerning its physical parameters. To limit the amount of free parameters in the radiative transfer models, we fixed several parameters. The spectral type of the central star of Frosty Leo has been estimated to be near K7III or II (Mauron et al. 1989). The effective stellar temperature $T_{\star}$ which corresponds to this spectral type is $\sim 3750 \mathrm{~K}$ (e.g. Robinson et al. 1992, and references therein). Such a low temperature is consistent with a negative detection of $\mathrm{H}_{2}$ emission lines in this object (e.g. Davis et al. 2005). There is no evidence for the presence of visible binary companions (Lopez et al. 2001, and our result shown in Fig. 1). Therefore, our model assumes a single radiation source with a black-body spectrum with an effective temperature of $3750 \mathrm{~K}$. The distance to Frosty Leo is somewhat uncertain. A lower limit of $1.0 \mathrm{kpc}$ was derived from the upper limit of the IRAS $10 \mu \mathrm{m}$ flux of $0.28 \mathrm{Jy}$, and the upper limit was estimated to be $4.3 \mathrm{kpc}$ assuming a spectral type of K7III $\left(M_{\mathrm{V}}=-0.4\right)$ and zero extinction (Mauron et al. 1989). The bolometric luminosity $L_{\star}$ has been estimated to be $250 D^{2} L_{\odot}$ (Rouan et al. 1988) and $300 D^{2} L_{\odot}$ (Forveille et al. 1987), where $D$ is the distance to Frosty Leo in kpc. The luminosity of a post-AGB star should be $>3000 L_{\odot}$ (e.g. Langill et al. 1994). Taking into account the uncertainty of the distance, we adopt the higher luminosity of $300 D^{2} L_{\odot}$ in our calculations. With this assumption, the uncertainty of the distance does not affect the SED.

Although detailed studies of the dust grain mineralogy in Frosty Leo have not been reported before, models of silicate cores with a water ice mantle have been widely assumed (Robinson et al. 1992; Lopez et al. 2001). In our models, we use the refractive indices of astronomical silicates (Draine 1985). L-band spectra (Rouan et al. 1988; Geballe et al. 1988; Hodapp et al. 1988) and a far-infrared spectrum (Omont et al. 1990) show the water ice absorption feature at $3.1 \mu \mathrm{m}$ and the emission features at 44 and $62 \mu \mathrm{m}$, respectively. Due to the similarity of the $3 \mu \mathrm{m}$ water ice absorption feature with the one of $\mathrm{OH} 231.8+4.2$ (Smith et al. 1988) and the presence of the $62 \mu \mathrm{m}$ emission feature, the carrier is thought to be pure $\mathrm{H}_{2} \mathrm{O}$ in crystalline form (Omont et al. 1990). We use refractive indices of water ice presented by Bertie et al. (1969). For $\lambda<1.25 \mu \mathrm{m}$, we assume a constant value of $n=1.301+0.0 i$, which is the value for $\lambda=1.25 \mu \mathrm{m}$. The fraction of water ice coat has been estimated to be between 50 and $70 \%$ in volume (e.g. Omont et al. 1990; Dougados et al. 1990).

Submicron-sized grains of $0.2-0.7 \mu \mathrm{m}$ have been predicted (Geballe et al. 1988; Hodapp et al. 1988) from conjectures that the $3.1 \mu \mathrm{m}$ band water ice absorption feature of Frosty Leo is similar to the one of $\mathrm{OH} 231.8+4.2$. Optical to NIR polarization analyses have estimated 0.1-2.0 $\mu \mathrm{m}$ radii (Dougados et al. 1990; Scarrott \& Scarrott 1994). On the other hand, SED and image fitting have suggested a $0.1-5.0 \mu \mathrm{m}$ size distribution (Lopez et al. 2001). In our modeling, we examine several grain models which are suggested from the observations, and optimize the parameters.

Several model geometries have been proposed to explain the bipolar CSE around evolved stars (e.g. Kahn \& West 1985; Meixner et al. 1999; Lopez et al. 2000; Men'shchikov et al. 2002; Ueta et al. 2003). We use the Kahn \& West-type geometry, which can reproduce the appearance of PPNs and PNs fairly well using a comparatively small number of free parameters (Kahn \& West 1985). The dust density distribution is given by

$\rho(r, \theta)=n_{0}\left(\frac{r}{R_{\text {in }}}\right)^{-\beta}\left(1+\epsilon \sin ^{\gamma} \theta\right)$.

Figure 4 shows a cross section of this geometry. The dust density distribution decreases with increasing radius, following the $r^{-\beta}$ power law. From the equatorial plane to the polar direction, the dust density drops by a factor of $(\epsilon+1)$ with an index of the latitudinal density gradient of $\gamma$. In our modeling, we adopt a fixed value of the outer radius $R_{\text {out }}$ of $40000 R_{\star}\left(7^{\prime \prime} .7\right)$, which is about the apparent size of the bipolar lobes detected in an HST optical image of Frosty Leo (Sahai et al. 2000).

\subsubsection{Models with a single grain species}

We first performed modeling with a single grain species and attempted to reproduce the observed SEDs and the total intensity images. We optimized the grain parameters within the following ranges: $a_{\min }$ of $0.005,0.01,0.02,0.05$, and $0.1 \mu \mathrm{m} ; a_{\max }$ of 0.5 , $1.0,2.0,5.0$, and $10.0 \mu \mathrm{m}$; the power-law index of the size distribution $p$ of 2.0 to 5.0 with increments of 0.5 , and the volume fraction of water ice $f_{\mathrm{m}}$ of 50,60 , and $70 \%$. For the above 450 grain models, we obtained SEDs and images with a geometry similar to the one adopted by Lopez et al. (2001) and investigated the dependence of the model results on the optical properties. The model results are quite insensitive with respect to the 


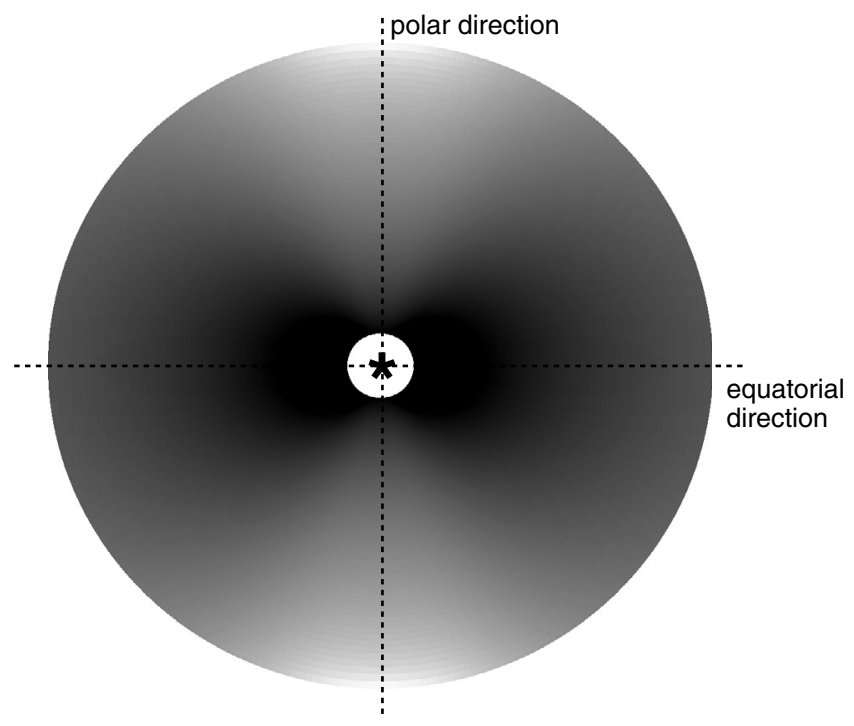

Fig. 4. Schematic representation of the two-dimensional dust density distribution of Frosty Leo. The cut direction is perpendicular to the equatorial plane. The density is high in the equatorial region and low in the polar region. See text for further details.

value of $a_{\min }$. Thus, we adopted a fixed value of $a_{\min }=0.005 \mu \mathrm{m}$ in our further models. The fitting was done by eye, taking into account the facts described below. Grains with maximum grain sizes $a_{\max }<1.0 \mu \mathrm{m}$ or a power-law index $p \gtrsim 4.0$ produce a result where the central star feature is almost obscured in the $H$ band if the optical depth is chosen to fit better in the $K$ band. This happens because the wavelength dependence of the extinction cross sections in the optical and NIR is too strong. On the other hand, grains with $a_{\max }>5 \mu \mathrm{m}$ and $p \lesssim 3.0$ produce a dust lane that is too weak and water ice emissions that are too shallow at $44 \mu \mathrm{m}$ and $62 \mu \mathrm{m}$, although the SED in the optical and NIR fits better. Thus, we have chosen a grain model with average parameter values of $a_{\max }=2 \mu \mathrm{m}$ and $p=3.5$, in agreement with Lopez et al. (2001). With respect to the water ice fraction, we found that a value of $f_{\mathrm{m}}=70 \%$ fits the optical depth of water ice at $3 \mu \mathrm{m}$ well with this grain model. The adopted opacities are plotted in Fig. 5a. Scattering dominates at $\lambda<6 \mu \mathrm{m}$ (albedo $\omega \sim 0.9$ ), while absorption dominates at $\lambda>6 \mu \mathrm{m}$. Absorption features attributed to water ice and silicates are present at $3.1 \mu \mathrm{m}$, $44 \mu \mathrm{m}$, and $62 \mu \mathrm{m}$, and $10 \mu \mathrm{m}$ and $18 \mu \mathrm{m}$, respectively.

With the above grain model, we searched for the geometry parameters to provide a good fit in the examined parameter sets. The ranges of the parameter values are: pole-to-equator density ratio $(\epsilon+1)$ of 5,10 , and 20; latitudinal density gradient $\gamma$ of $5,10,15,20$, and 30 ; power-law index of the radial density gradient $\beta$ of 2, 3, and 4 (Dougados et al. 1990; Lopez et al. 2001); inner radius $R_{\text {in }}$ of $0{ }^{\prime} 7,1^{\prime \prime} .0$, and $1^{\prime \prime} .4$ (cf. $\sim 1^{\prime \prime}(0$, Dougados et al. 1990); and optical depth $\tau_{2.2}$ at $2.2 \mu \mathrm{m}$ towards the equatorial plane of 5 to 10 with $\Delta \tau_{2.2}=1$ (cf. $\tau=10$ 15 at $1.0 \mu \mathrm{m}$, Robinson et al. 1992; Lopez et al. 2001). From SED fitting, we derived $\tau_{2.2}=6$ and $R_{\text {in }}=5200 R_{\star}$ for our selected model. From image fitting, we obtained values of 8,10 , and 3 for $(\epsilon+1), \gamma$, and $\beta$, respectively. We set the viewing angle $\theta$ to $90^{\circ}$ (edge-on view), because we found that asymmetry between the two bipolar lobes becomes too strong for viewing angles $\lesssim 85^{\circ}$ with this model geometry. Figure $5 \mathrm{~b}$ compares the model SED to the observations. A calibration of the interstellar extinction is not applied in the observed SED since it is negligible due to Frosty Leo's high Galactic latitude of $b=+42.7^{\circ}$ (e.g. Unavane et al. 1998). The SED of Frosty Leo shows a
Table 1. Parameters of our selected 2-D radiative transfer models of Frosty Leo with a single grain species model.

\begin{tabular}{|c|c|c|}
\hline Parameters & Adopted values & Comment $^{[1]}$ \\
\hline \multicolumn{3}{|c|}{ stellar properties $^{[2]}$} \\
\hline$T_{\text {eff }}$ & $3750 \mathrm{~K}$ & adopted $^{[3]}$ \\
\hline$L_{\star}$ & $300 D^{2} L_{\odot}$ & adopted $^{[4]}$ \\
\hline$R_{\star}^{\star}$ & $2.86 \times 10^{12} \mathrm{~cm}$ & calculated \\
\hline$\alpha_{\star}$ & $0.191 \mathrm{mas}$ & calculated \\
\hline \multicolumn{3}{|c|}{ model geometry (common) } \\
\hline$R_{\text {in }}$ & $5200 R_{\star}$ & derived \\
\hline$R_{\text {out }}$ & $40000 R_{\star}$ & adopted \\
\hline \multicolumn{3}{|c|}{ SED + image fit model } \\
\hline$\beta$ & 3 & $2-3$ \\
\hline$\gamma$ & 10 & $5-10$ \\
\hline$(\epsilon+1)$ & 8 & $8-10$ \\
\hline$\tau_{2.2}^{[5]}$ & 6 & $6-7$ \\
\hline$a_{\min }^{2.2}$ & $0.005 \mu \mathrm{m}$ & $0.005-0.1$ \\
\hline$a_{\max }$ & $2.0 \mu \mathrm{m}$ & $2.0-5.0$ \\
\hline$p$ & 3.5 & $3.0-3.5$ \\
\hline$f_{\mathrm{m}}$ & $70 \%$ & $60-70$ \\
\hline \multicolumn{3}{|c|}{ image + polarization fit model } \\
\hline$\beta$ & 2 & $2-3$ \\
\hline$\gamma$ & 10 & $5-10$ \\
\hline$(\epsilon+1)$ & 10 & $8-10$ \\
\hline$\tau_{2.2}^{[5]}$ & 6 & $4-6$ \\
\hline$a_{\min }$ & $0.005 \mu \mathrm{m}$ & fixed \\
\hline$a_{\max }$ & $0.7 \mu \mathrm{m}$ & $0.6-0.7$ \\
\hline$p$ & 3.5 & fixed \\
\hline$f_{\mathrm{m}}$ & $70 \%$ & $60-70$ \\
\hline
\end{tabular}

${ }^{1}$ Ranges give the uncertainty of the corresponding model parameters, ${ }^{2}$ common parameters for all of our models, ${ }^{3}$ Robinson et al. (1992), ${ }^{4}$ Forveille et al. (1987), ${ }^{5}$ towards the equatorial plane.

double peak, which is frequently seen in PPNs with a detached shell (e.g. Kwok 1993). The shorter (optical to NIR) and longer (MIR to FIR) wavelength peaks are dominated by scattered light and thermal emission from dust, respectively. The dust temperature at the inner boundary is obtained to be $\sim 63 \mathrm{~K}$ from our modeling, suggesting that dust grains in the entire nebula may be covered with a water ice mantle. The model SED for $\lambda \geq 2 \mu \mathrm{m}$, including the absorption and emission features at 3.1, 44, and $62 \mu \mathrm{m}$, is in good agreement with the observations. On the other hand, the flux in the optical to NIR of our model is lower than observed. This is probably also due to a stronger wavelength dependence of the scattering cross section of our dust model. The parameters of our selected model with a single grain species are listed in Table 1 . Figures $5 \mathrm{c}$ and d show the $H$ - and $K$-band intensity images, respectively. The dust lane, bipolar lobes, and the central star feature are reproduced. The latitudinal dust density gradient $\gamma$ and the ratio of pole-to-equator dust density $(\epsilon+1)$ affect the bipolar appearance in the total intensity image. In our studies, a larger $\gamma$ produces an elongation of the flux peak in the bipolar lobes that is too pronounced, and a larger $(\epsilon+1)(>20)$ results in a nebula flux that is too low. A lower optical depth $\left(\tau_{2.2}=5\right)$ fits the SED in the optical and NIR better, but the feature of the dust lane becomes too weak. A higher optical depth $\left(\tau_{2.2}>8\right)$ results in a more distinct dust lane, but the fluxes in the optical and NIR become too low and the central star is too obscured. In our selected model, the equatorial dust lane is seen within only 1 '. 5 from the central source, while the apparent size in our observed images (see Figs. 1 and 2 ) is $\sim 3^{\prime \prime}$. Figures 5 e and $f$ show the degree of polarization images with polarization vector lines in the $H$ and $K$ bands, respectively. We found that 

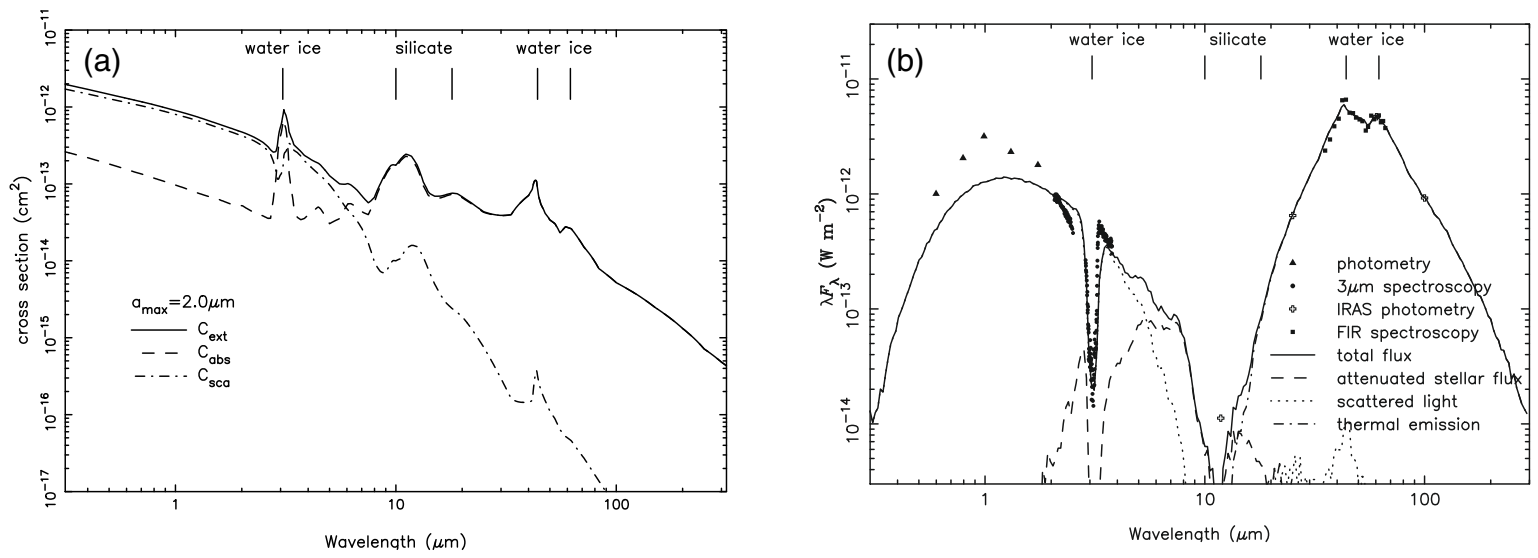

Wovelenath $(\mu \mathrm{m})$


Fig. 5. Results of our selected 2-D radiative transfer model of Frosty Leo with a single grain species $\left(a_{\max }=2.0 \mu \mathrm{m}\right)$ based on SED and intensity image fitting. a) Cross sections as a function of wavelength for the grain species adopted in our radiative transfer calculations. The grain model has $a_{\max }=2.0 \mu \mathrm{m}$ and $f_{\mathrm{m}}=70 \%$. b) Comparison of model SED to observations. Filled triangles, filled circles, open crosses, and filled squares denote results from aperture photometry (Rouan et al. 1988), $3 \mu \mathrm{m}$ spectroscopy (Geballe et al. 1988), IRAS photometry, and FIR spectroscopy (Omont et al. 1990), respectively. Solid, dashed, dotted, and dashed-dotted lines denote the total flux, attenuated stellar flux, flux of scattered light, and thermal emission by dust, respectively. c)-f) are the $H$ - and $K$-band intensity images and the $K$-band polarization image, respectively. The model images are convolved with a Gaussian PSF with a 0!'2 FWHM, which corresponds to the PSF of our observations. The viewing angle is $90^{\circ}$ (edge-on view). The unit of the intensity image is same as that of the observations (i.e., $\mathrm{W} \mathrm{m}^{-2} \mu \mathrm{m}^{-1} \operatorname{arcsec}^{-2}$ ). The same color scales to the observations are used in the corresponding images. 

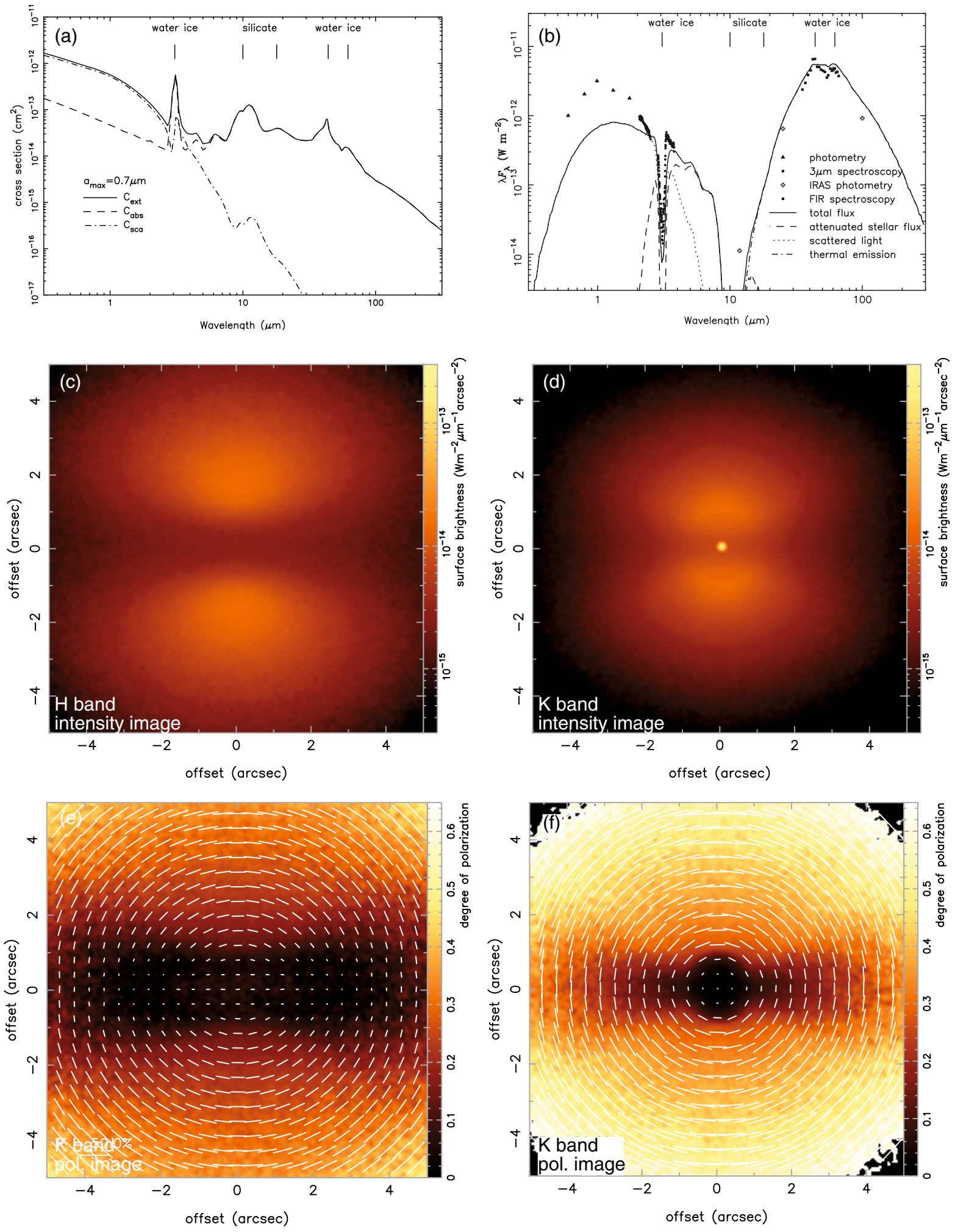

Fig. 6. Same as Fig. 5 but for the single grain species model $\left(a_{\max }=0.7 \mu \mathrm{m}\right)$ based on a fit of intensity and polarization images.

the degree of polarization reaches, at most, $\sim 27 \%$ in the entire nebula, while it is $40-55 \%$ according to our observations. It is known that the larger the grain size, the lower the polarization of the singly scattered light (e.g. Fig. 2 in the paper by Fischer et al. 1994). Therefore, the too low polarization in our selected model suggests that the grain size is too large for grains in the bipolar nebula.
In a second step, we attempted fits of the intensity images and the polarization images. We examined grain parameters of $a_{\max }$ between $0.5 \mu \mathrm{m}$ and $1.0 \mu \mathrm{m}$ in increments of $0.1 \mu \mathrm{m}$ and $f_{\mathrm{m}}$ of 50,60 , and $70 \%$. The size distribution power-law index $p$ was set to 3.5 . The total intensity images and the degree of polarization images were generated for models with the geometry parameter sets used for the previous SED fitting. We focused on the $K$ band because we could not find a solution which fits 
both the $H$ and $K$ bands simultaneously. The parameters of the selected model are: $a_{\max }=0.7 \mu \mathrm{m}, f_{\mathrm{m}}=70 \%, \tau_{2.2}=6, \beta=2$, $(\epsilon+1)=10$, and $\gamma=10$, and they are summarized in Table 1 . If the model is optimized in the $H$ band, an $a_{\max }$ of $0.6 \mu \mathrm{m}$ and an optical depth $\tau_{2.2}$ of $4-5$ are favored. The grain opacities are presented in Fig. 6a. The opacities have a stronger wavelength dependence compared to the $2 \mu \mathrm{m}$ grains, particularly in the NIR. Figure 6 shows the model results. The distinct bipolar appearance of Frosty Leo is reproduced in the intensity image and the polarization disk is visible. The difference of the bipolar appearance from the previous SED fitting model can be explained with the scattering properties of the modeled dust. The scattering asymmetry parameters $g$ of the $2.0 \mu \mathrm{m}$ and the $0.7 \mu \mathrm{m}$ grain models are 0.61 and 0.38 at $\lambda=2.2 \mu \mathrm{m}$, respectively. In the case of large grains, photons propagate through the equatorial plane by changing the direction of scattered light only slightly. On the other hand, small grains change the direction more than large grains and photons can escape from the equatorial plane more easily. Thus, in models with small grains, a lower flux in the equatorial plane is obtained, which results in a distinct dust lane. Furthermore, small grains absorb optical and NIR photons more efficiently than large grains. Although the difference of the dust albedos between the $0.7 \mu \mathrm{m}$ grain model $(0.83$ at $\lambda=2.2 \mu \mathrm{m})$ and the $2.0 \mu \mathrm{m}$ grain model $(0.89$ at $\lambda=2.2 \mu \mathrm{m})$ is not so large, this effect could, to some extent, reduce the light propagating through the equatorial plane more than the light propagation through the polar region. The degree of polarization reaches up to $\sim 40 \%$ and $\sim 60 \%$ in the $H$ and $K$ bands, respectively, which is in good agreement with our observations. However, the SED fit becomes worse: lower fluxes in the optical to NIR and slightly higher fluxes in FIR. The former is due to a too strong wavelength dependence of the dust opacity. It can also explain a too low flux in the SED and a too faint central star feature compared to the flux peaks in the NS lobes in the $H$ band intensity image. The higher FIR fluxes are due to too much FIR emission by small grains. Furthermore, the appearance of the polarization disk in the polarization maps has a too strong wavelength dependence compared to the observations. Since a polarization disk appears towards the optically thick region, the too strong wavelength dependence suggests that the wavelength dependence of the grain opacity is too large, implying that the grain size near the equatorial region is too small.

\subsubsection{Models with two grain species}

Our models described in the previous section suggest that the SED fitting favors large grains, while the polarization properties in the bipolar nebulosity require small grains. In order to solve such contradictory requirements, we examined models with two different grain species in different regions of the Frosty Leo nebula. The SED in the optical and NIR is mainly dominated by flux from regions close to the central star. The central star feature is clearly visible, and the appearance of the polarization disk is similar between the $H$ and $K$ bands. On the other hand, a high polarization is detected in the bipolar lobes. Thus, we assumed large grains in the high-density region near the equatorial plane (disk region) and small grains in the lobes. Furthermore, we made the disk geometrically thin and viewed the system not quite edge-on in order to reproduce the prominent stellar feature and the distinct dust lane simultaneously.

For simplicity, we used the same geometry for the bipolar lobes as in the polarization fit model, but different parameters for the inner disk. The disk is placed in the region with radii between $R_{\text {in }}$ and $R_{\text {disk }}$ and latitudes between $-\theta_{\text {disk }}$ and

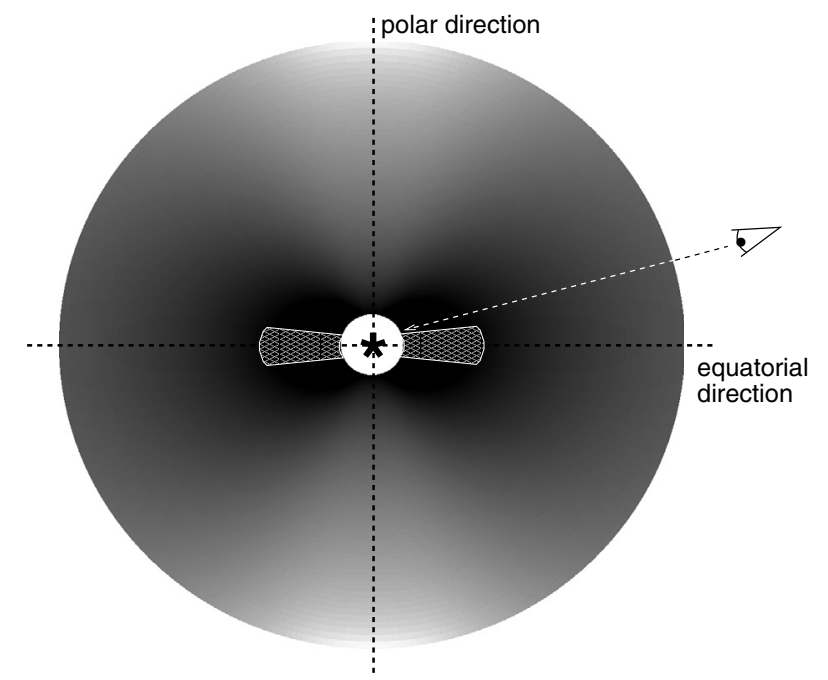

Fig. 7. Schematic representation of the dust shell of the two grain species model. A geometrically thin disk is placed at latitudes between $-\theta_{\text {disk }}$ and $+\theta_{\text {disk }}$ at radial positions $R \leq R_{\text {disk }}$ inside the Kahn \& Westtype dust shell. In this disk region, grains have a size distribution from $0.005 \mu \mathrm{m}$ to $2.0 \mu \mathrm{m}$. On the other hand, small grains with sizes between $0.005 \mu \mathrm{m}$ and $0.7 \mu \mathrm{m}$ are placed in the bipolar lobes.

$+\theta_{\text {disk }}$. The density structure has a power-law density distribution: $\rho(r, \theta)=A n_{0}\left(r / R_{\text {in }}\right)^{-\beta}$, cf. Eq. (1). A cartoon of the dust density distribution of this model geometry is presented in Fig. 7. The model parameters and their ranges are: disk radii $R_{\text {disk }}$ of 8000,10000 , and $30000 R_{\star}$; disk thicknesses $\theta_{\text {disk }}$ of $2^{\circ}$ to $5^{\circ}$ with increments of $1^{\circ}$; disk density factors $A$ of $10,20,50$, and 100; optical depths towards the equatorial plane of $7,10,20$, and $50 ; a_{\mathrm{max}}$ and $f_{\mathrm{m}}$ values of grains in the disk of $1.0,2.0,5.0$, and $10.0 \mu \mathrm{m}$ and 50,60 , and $70 \%$, respectively; and an $f_{\mathrm{m}}$ of grains in the nebula of 50,60 , and $70 \%$. We fixed the power-law in$\operatorname{dex} \beta$ of the disk and the maximum size of small grains in the lobes to 2 and $0.7 \mu \mathrm{m}$, respectively. For these 5832 models, we calculated SEDs for viewing angles between $80^{\circ}$ and $90^{\circ}$ with increments of $1^{\circ}$. Then, we generated Stokes images and chose a proper model. The parameters of the selected model are summarized in Table 2. We also estimated the dust mass of the disk, the mass-loss rate at the inner radius, and the envelope mass including the disk to be $2.85 \times 10^{-3} M_{\odot}, 8.97 \times 10^{-3} M_{\odot} \mathrm{yr}^{-1}$, and $4.23 M_{\odot}$, respectively, assuming a distance of $3 \mathrm{kpc}$ (Sahai et al. 2000), an expansion velocity of $25 \mathrm{~km} \mathrm{~s}^{-1}$ (Forveille et al. 1987; Castro-Carrizo et al. 2005), and a gas-to-dust mass ratio for oxygen-rich evolved stars of 160 (Knapp 1985).

Figure 8 presents the adopted dust opacity, the model results of the SED, the intensity images, and the polarization images. With respect to the dust opacity, the only difference between the single-grain species model and the two-grain species model is a shallower water ice feature in the latter one because of a lower $f_{\mathrm{m}}$ value. The model SED is in good agreement with the observations, except in the optical. Despite adopting a lower $f_{\mathrm{m}}$ value, the water ice absorption feature is deeper in this model than in the SED fit model. This happens because smaller grains, which are placed in the lobes in the two-grain species model, produce a deeper absorption feature, as can be seen from a comparison of Figs. 5a and $8 \mathrm{~b}$. In the intensity images, a dust lane and a prominent stellar feature are seen in the $H$ and $K$ bands, respectively. The degree of polarization in the nebula of this model reaches $P_{H}=30 \%$ and $P_{K}=60 \%$, which is in basic agreement with the observed polarizations of $P_{H}=35-50 \%$ and $P_{K}=40-55 \%$. Furthermore, compared to the model results with the $0.7 \mu \mathrm{m}$ 
Table 2. Parameters of our selected 2-D radiative transfer model of Frosty Leo with two grain species.

\begin{tabular}{lcl}
\hline \hline Parameters & Adopted values & Comment $^{[1]}$ \\
\hline \multicolumn{3}{c}{ bipolar lobe } \\
$\gamma$ & 2 & adopted \\
$(\epsilon+1)$ & 10 & adopted \\
$a_{\max }$ & 10 & adopted \\
$f_{\mathrm{m}}$ & $0.7 \mu \mathrm{m}$ & adopted \\
\hline \multicolumn{4}{c}{$50 \%$} & $50-60$ \\
$\beta$ & disk \\
$A$ & 2 & adopted \\
$a_{\max }$ & 50 & $20-50$ \\
$f_{\mathrm{m}}$ & $2.0 \mu \mathrm{m}$ & $2.0-5.0$ \\
$\theta_{\text {disk }}$ & $50 \%$ & $50-60$ \\
$R_{\text {disk }}$ & $2^{\circ}$ & $2^{\circ}-3^{\circ}$ \\
\hline \multicolumn{4}{c}{ others } \\
$\tau_{2.2}^{[2]}$ & 20 & $8000-10000$ \\
$\tau_{2.2}^{[3]}$ & 7.5 & $10-20$ \\
$\tau_{1.65}^{[3]}$ & 12.5 & calculated \\
$\theta_{\mathrm{v}}$ & $87^{\circ}$ & calculated \\
$M_{\text {disk }}^{[4]}$ & $2.85 \times 10^{-3} M_{\odot}$ & dust mass \\
$\dot{M}^{[4]}$ & $8.97 \times 10^{-3} M_{\odot} \mathrm{yr}^{-1}$ & at $R_{\text {in }}$ \\
$M_{\mathrm{env}}^{[4]}$ & $4.23 M_{\odot}$ & includes the disk \\
\hline
\end{tabular}

${ }^{1}$ Acceptable ranges, ${ }^{2}$ towards the equatorial plane ${ }^{3}$ towards the observer, ${ }^{4}$ assuming a distance of $3 \mathrm{kpc}$ (Sahai et al. 2000), an expansion velocity of $25 \mathrm{~km} \mathrm{~s}^{-1}$ (Forveille et al. 1987; Castro-Carrizo et al. 2005), and a gas-to-dust mass ratio for oxygen-rich evolved stars of 160 (Knapp 1985).

grains, the difference of the appearance of the polarization disk between the $H$ and $K$ bands becomes smaller in the two grain species model. Our model introducing a second grain population can provide a better solution, although a significant improvement is not obtained.

The difficulty in modeling the Frosty Leo nebula can be summarized as follows. The facts that (1) the SED fit predicts a low wavelength dependence of the dust opacity; (2) the central star feature is seen in the optical to NIR; and (3) the appearance of the polarization disk is similar between the $H$ and $K$ bands seem to favor large grains in the equatorial region. In contrast, such grains makes it difficult to produce a distinct dust lane. The above contradiction is hard to solve assuming a two-dimensional geometry and spherical grains. It would require a three-dimensional geometry or maybe some other model properties, such as non-spherical grains in the nebula. The disk would have an inhomogeneous density distribution in the azimuthal direction and, by chance, the optical depth should be low towards the observer but high enough in other directions to produce a distinct dust lane. But, we would like to stress that models with a small disk radius are not reliable because they cause a too high flux around 20-40 $\mu \mathrm{m}$. Large grains (e.g. $a_{\max }>5 \mu \mathrm{m}$ ) may exist in the line of sight towards the central star but not enough to smooth up the 44 and $62 \mu \mathrm{m}$ water ice emission feature. The possibility of a fluffy structure of grains in the Frosty Leo nebula will be clarified if the detailed optical properties are investigated (e.g. Wright et al. 1987; Kozasa et al. 1992, 1993; Voshchinnikov et al. 2005; Min et al. 2006; Voshchinnikov \& Henning 2008).

Concerning the polarization properties, the observed polarization vector alignment could not be reproduced in our modeling. We examine three mechanisms which have been proposed so far to explain such a polarization vector alignment: (1) the PSF smoothing effect (e.g. Piirola et al. 1992; Gledhill 2005);
(2) multiple scattering at the boundaries of a geometrically thin disk (Bastien \& Ménard 1988); and (3) linear dichroism by aligned, non-spherical grains (e.g. Hough et al. 1989; Whitney $\&$ Wolff 2002). The first mechanism is ruled out because the two bipolar lobes are sufficiently separated $\left(\sim 2^{\prime \prime}\right)$ compared to the PSF size of $\sim 0$ !' 2 , and the prominent central star produces strong unpolarized light. Under such conditions, the polarization could not be as high as $\sim 13 \%$ near the central star. The second mechanism, multiple scattering at the boundaries of a geometrically thin disk, is also unlikely. In our two-grain species model, we use a geometrically thin, optically thick disk, which is a potential source to produce the polarization alignment via this mechanism. However, our model does not show a high polarization in the disk because the disk is filled with large (micron-sized) grains, which is very likely in the real Frosty Leo disk. Because our two-grain species model with a disk shows a polarization disk with nearly unpolarized light, the multiple-scattering mechanism is probably not relevant for Frosty Leo. However, we emphasize that our observations do not rule out the possibility of the presence of the disk. For reasons outlined above, only the third case of aligned non-spherical grains remains as the most plausible mechanism to explain the observed polarization vector alignment. In previous observations, the degree of linear polarization had a higher value at shorter wavelengths (e.g. Hough et al. 1989), which was probably due to the wavelength dependence of dichroic absorption. On the other hand, our observations show a nearly constant polarization $(P \sim 12-13 \%)$. This can be explained with a weak wavelength dependence of the dust opacity due to large grains in this region. Measurements of circular polarization or NIR and $3 \mu \mathrm{m}$-band spectropolarimetry would help to investigate this issue in more detail.

\section{Discussion}

We tried to find a model which is able to reproduce (1) the observed SED; (2) the intensity images; and (3) the polarization images of Frosty Leo. From our modeling, we conclude that at least two grain species exist at different locations in the nebula. On the other hand, a previous optical imaging polarimetry suggested a different grain model. Scarrott \& Scarrott (1994) obtained polarimetric images in the $B, V, R$, and $I$ bands. The degree of polarization at the ansae $10^{\prime \prime}-15^{\prime \prime}$ away from the central star and in the central hourglass-shaped lobes in the $R$ band were found to be $36-60 \%$ and $20-30 \%$, respectively. From their grain modeling, they found that a grain model with sizes of $0.09 \leq a \leq 2.0 \mu \mathrm{m}$ and a 3.5 power-law index of the size distribution produces too low polarization and that a grain model with a 5.2 power-law index fits the polarization well. Although their $a_{\max }$ value is the same as ours, their power-law index is sufficiently larger. The dust properties of such a grain model are characterized by the $a_{\min }(\sim 0.1 \mu \mathrm{m})$ value rather than the $a_{\max }$ value. With our two-grain species model, we obtained polarizations of $\leq 15 \%$ in the hourglass-shaped lobes in the $B, V, R$, and $I$ bands, where the $0.7 \mu \mathrm{m}$ grains determine the polarization. It is obvious that such a low polarization is due to too large grains in our models. However, taking into account the NIR polarizations of $35-60 \%$ in the $H$ and $K$ bands and also $\sim 30 \%$ in the $J$ band (Dougados et al. 1990), it could be possible that a region with small grains $\left(a_{\max } \sim 0.1 \mu \mathrm{m}\right)$ surrounds an inner region with sub-micron sized grains and probably extends $\sim 15^{\prime \prime}$ from the central star.

Based on observations of mass-losing evolved stars, a large fraction of dust is expected to have a $0.3 \mu \mathrm{m}$ or smaller size (Dominik et al. 1989; Jura 1996). It could be possible that grains which formed in the course of the AGB mass loss survived 

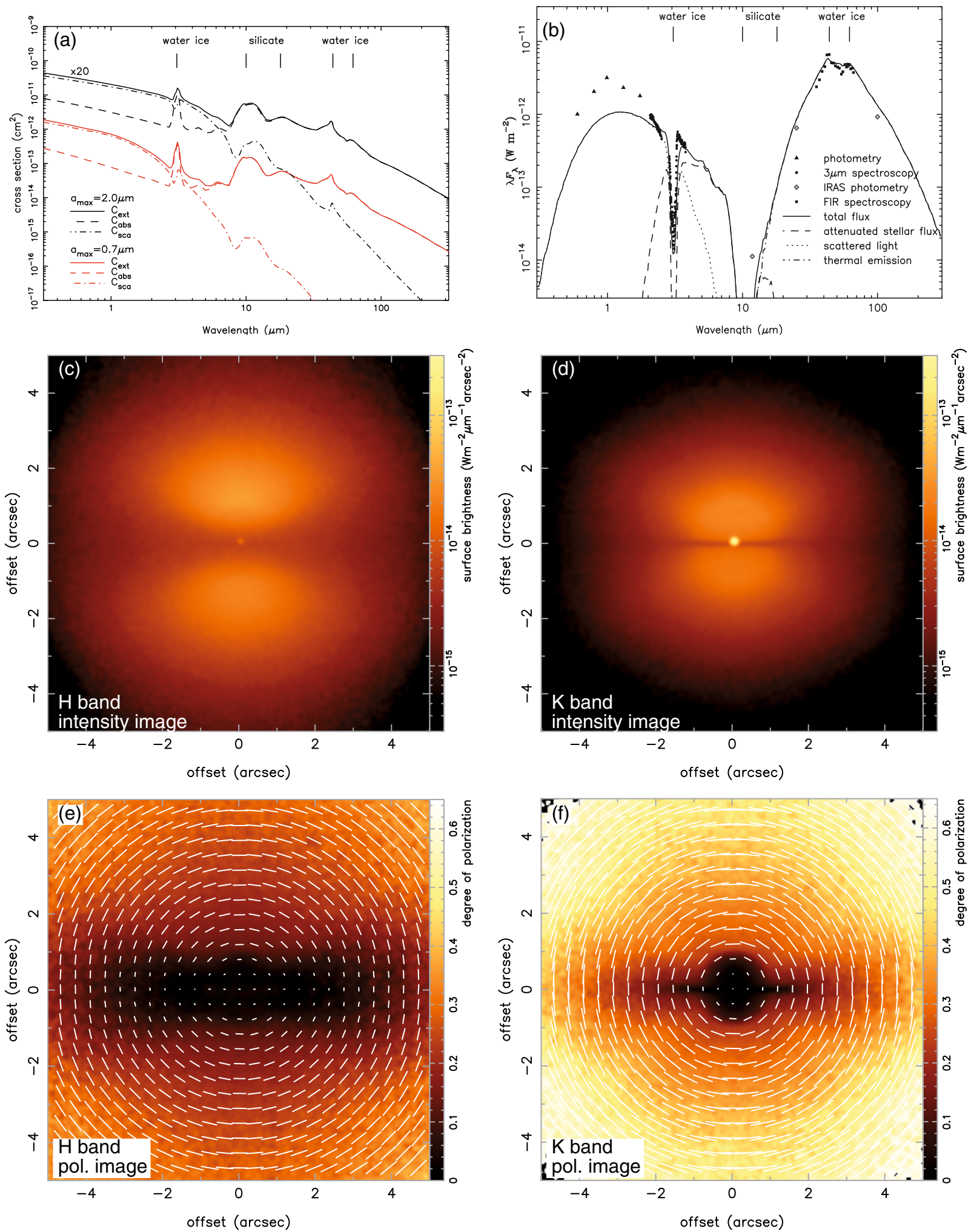

Fig. 8. Results of our selected 2-D radiative transfer model of Frosty Leo with two grain species. a) Comparison of the model SED to observations. b) Cross section as a function of wavelength for grains with $a_{\max }=2.0 \mu \mathrm{m}$ (black) and with $a_{\max }=0.7 \mu \mathrm{m}$ (red). For the sake of clarity, the opacity for $a_{\max }=2.0 \mu \mathrm{m}$ is multiplied by 20. c)-f) are the intensity and polarization images in the $H$ and $K$ bands. The model images are convolved with a Gaussian PSF with a $0 ! 2$ FWHM. The viewing angle is $87^{\circ}$; i.e., the upper (northern) lobe is tilted towards the observer (see also Fig. 7).

without growth until some time in the recent past. Then, grain growth started at the very end of the AGB phase in the Frosty Leo nebula. The evidence for the presence of large grains $(a \gtrsim$ $100 \mu \mathrm{m})$ has been reported in some PPNs such as the Red Rectangle (Jura et al. 1997), AFGL 2688 (Jura et al. 2000a), and IRAS 18276-1431 (Sánchez Contreas et al. 2007). If the dust is ejected in the expanding stellar wind, it is unlikely that such large grains grow in the nebula. Therefore, the idea of a long-lived disk or reservoir has been proposed (Jura et al. 2000b; Yamamura et al. 2000). According to this idea, grains are trapped in the disk (circumbinary disk or circumcompanion disk) and orbit around the central star for a long time span. Dust can grow 
in size due to grain-grain collision. In fact, a Keplerian rotating motion with a nearly zero expansion velocity has been detected in the Red Rectangle (Bujarrabal et al. 2003). The inner radius of the dust shell is staying at the dust sublimation radius of $\sim 14 \mathrm{AU}$, although the central star evolved as the stellar temperature became as high as $T_{\star} \sim 7700 \mathrm{~K}$ (Men'shchikov et al. 2002). Such a small inner radius seems to be commonly detected in PPNs with a Keplerian motion (De Ruyter et al. 2006) and leads to obscuration of the central star. Furthermore, the FIR flux excess can be explained with large grains instead of the density structure (Jura et al. 2000a). On the other hand, such signatures are not seen in Frosty Leo. High resolution CO emission line observations show no indication of Keplerian rotating motions around the equatorial plane (Castro-Carrizo et al. 2005). Taking into account a large inner radius of $5200 R_{\star}(=1000 \mathrm{AU}$ at a distance of $3 \mathrm{kpc}$ ), the visible central star feature, and an intermediate velocity width $\Delta v_{\mathrm{CO}} \sim 50 \mathrm{~km} \mathrm{~s}^{-1}$ (Forveille et al. 1987), the inner part of the equatorial region is probably an expanding disk rather than a rotating disk. Such disk environments will play an important role with respect to the grain growth evolution. Further observations and modeling of other bipolar PPNs would help to provide new insights into the physical conditions for grain growth in the central disk.

\section{Conclusion}

We presented a diffraction-limited ( $0{ }^{\prime} 12$ resolution) $K^{\prime}$-band bispectrum speckle image and $H$ - and $K$-band AO polarimetric images of the PPN Frosty Leo. Our speckle image resolves clumpy structures in the bipolar lobes. We found no evidence of a binary companion of the central star. Our polarimetric data show three interesting polarization signatures. First, a polarization disk extending $\sim 5^{\prime \prime}$ along the dust lane. A low polarization in the dust lane indicates an equatorially enhanced density distribution (i.e. disk), which is responsible for the bipolar appearance of Frosty Leo. Second, an elongated region with low polarizations is seen along a PA of $-45^{\circ}$. This region is likely to be a tube-like structure with a low density, which is carved out by a jet labeled J1' in an HST optical image (Sahai et al. 2000). Third, polarization vectors in the dust lane $\sim 1^{\prime \prime}$ east of the central star are aligned. This could be due to aligned non-spherical grains.

In order to investigate the dust grain properties and the nebula structure of Frosty Leo, we performed 2-D radiative transfer modeling using the Monte Carlo code mcsim_mpi. From SED and intensity image fits, models with grain sizes between $0.005 \mu \mathrm{m}$ and $2.0 \mu \mathrm{m}$ are favored. However, such dust models result in too low polarization values in the bipolar lobes. On the other hand, our models using small grains with $0.005 \leq a \leq 0.7 \mu \mathrm{m}$ and $f_{\mathrm{m}}=70 \%$ are in agreement with the intensity image and the polarization, and are able to reproduce the distinct dust lane, the hourglass-shaped bipolar lobes in the $K$ band, and the degree of polarization in the lobes $\left(P_{H} \sim 40 \%\right.$ and $P_{K} \sim 60 \%$ ). In order to solve these contradictory results, i.e., the requirement of large grains from the SED fit, the appearance of the central star feature, the wavelength dependence of the appearance of the polarization disk, and small grains from the polarization fit, we derived models with two different grain sizes at different locations in the nebula. We placed $2.0 \mu \mathrm{m}\left(f_{\mathrm{m}}=50 \%\right)$ grains in a geometrically thin disk near the equatorial plane between latitudes $-2^{\circ}$ and $+2^{\circ}$, and $0.7 \mu \mathrm{m}$ grains $\left(f_{\mathrm{m}}=50 \%\right)$ in the lobes. With this model viewed at an angle of $87^{\circ}$, the observed SED, the distinct dust lane with a prominent central star feature, and also the high polarization in the nebula and the polarization disk are approximately reproduced. Our experiment illustrates that NIR multiple wavelength imaging polarimetry and radiative transfer modeling constraining by SED, intensity image, and polarization as well as treating multiple grain species are powerful technique to derive the dust grain properties (grain size) and the nebula geometry. From our modeling of Frosty Leo, we derived the dust disk mass, the inner radius of the disk, the mass-loss rate, and the total envelope mass including the disk mass to be $2.85 \times 10^{-3} M_{\odot}, 1000 \mathrm{AU}, 8.97 \times 10^{-3} M_{\odot} \mathrm{yr}^{-1}$, and $4.23 M_{\odot}$, respectively.

Acknowledgements. Based on data collected at the European Southern Observatory and at the Subaru observatory. We would also like to thank Dr. T. M. Gledhill for useful discussions on the radiative transfer code.

\section{Appendix A: Tests for Stokes images}

The mcsim_mpi code has been tested with respect to the SED, the dust temperature distribution, and the total intensity (Stokes I) images by comparing the results with those of other radiative transfer codes. Recently, mcsim_mpi was used to interpret the MIR spectro-interferometric data of the silicate carbon star IRAS 08002-3803 (Ohnaka et al. 2006). Here, we test our modification of the code to generate the Stokes images. As dust grains, we use astronomical silicates with a size distribution of $n(a) \propto a^{-3.5}$ and $0.005 \leq a \leq 0.25 \mu \mathrm{m}$. We assume monochromatic light at a wavelength of $2.2 \mu \mathrm{m}$. The extinction cross section $C_{\text {ext }}$, albedo $\omega$, and scattering asymmetry $g$-parameter at $2.2 \mu \mathrm{m}$ are $2.99 \times 10^{-14} \mathrm{~cm}^{2}, 0.292$, and 0.0703 , respectively. We apply a Kahn \& West-type dust density distribution (Kahn \& West 1985), as given by Eq. (1) and presented in Fig. 4. The geometry parameters are $\epsilon=9, \beta=2, \gamma=5$ with inner and outer radii of $100 R_{\star}$ and $10^{4} R_{\star}$, respectively. The stellar parameters are the effective temperature of $5000 \mathrm{~K}$ and the bolometric luminosity of $10^{4} L_{\odot}$. We modeled an optically thin case with $\tau_{2.2 \mu \mathrm{m}}=1$ towards the equatorial plane and an optically thick case with $\tau_{2.2 \mu \mathrm{m}}=10$ to see the differences of the nebula shape and the polarization signatures. Figure A.1 shows the results of the total intensity images in the upper panels and the polarization images with polarization vector lines in the lower panels (edge-on view).

In the optically thin case, the total intensity image shows a prominent spherical feature around the central star and an elliptically elongated nebula towards the equatorial direction. The intensity distribution in the nebula reflects the column density of the dust grains. The polarization map shows a centro-symmetric polarization vector pattern in the entire nebula, which is due to single scattering. The degree of polarization is very low close to the central star because of contamination by the unpolarized component of the direct light from the central star and gradually increases with increasing distance from the central star. We also find hourglass-shaped regions with slightly lower polarizations in polar direction. These are caused by less scattered light due to the lower grain density near the plane of the sky, which provides large polarizations (see also polarization diagrams in Fig. 2; Fischer et al. 1994).

In the optically thick case, the total intensity image shows bipolar lobes extending along the polar direction and a dust lane lying in the equatorial plane between the lobes. In the polarization map, a centro-symmetric vector pattern is still seen in the bipolar lobes because the nebula is optically thin in polar direction. We also see a polarization disk and polarization vector alignment along the equatorial plane. A remarkable thing is that the polarization contrast is reversed compared to the optically thin case because singly scattered light, which has a high polarization, is well attenuated (i.e., it undergoes subsequent interactions until it leaves the model envelope), and polarized light from various different PAs in the line of sight cancels the degree 

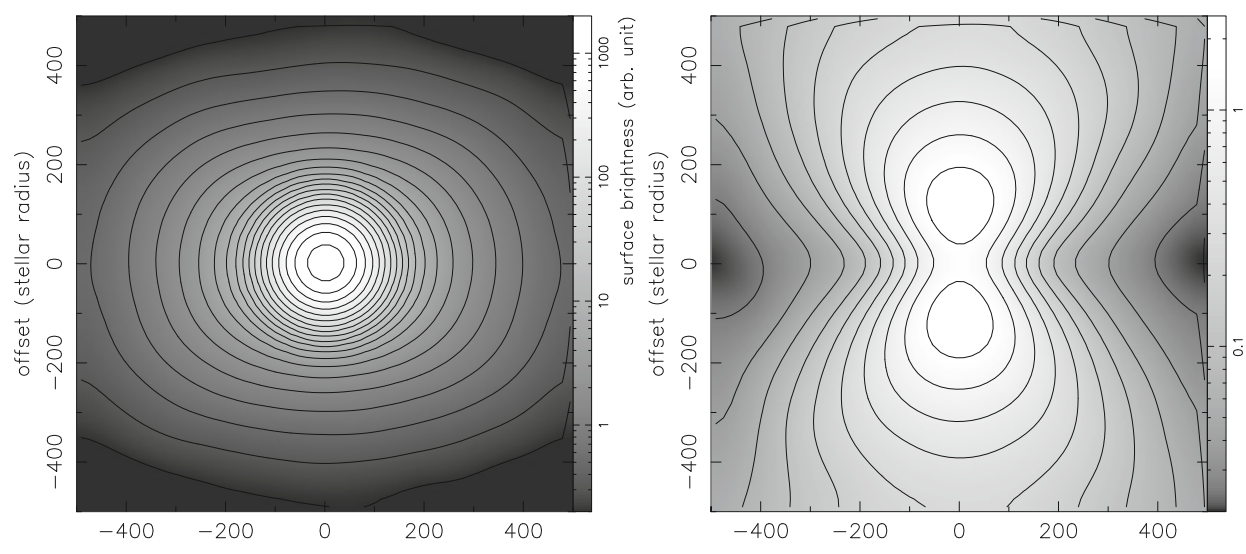

offset (stellar radius)

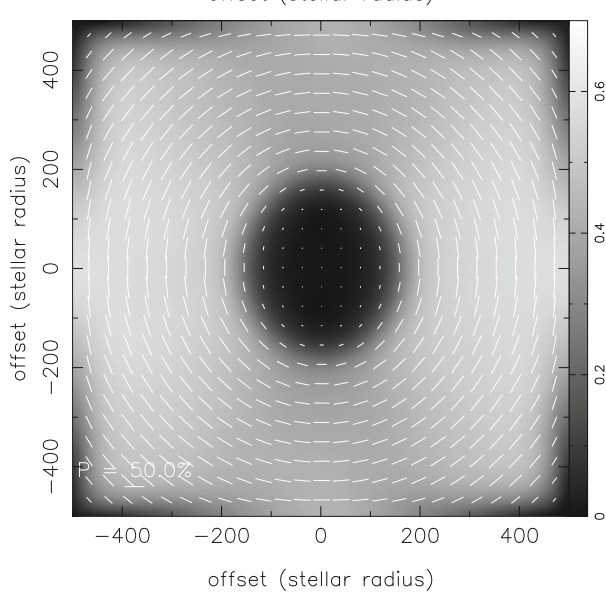

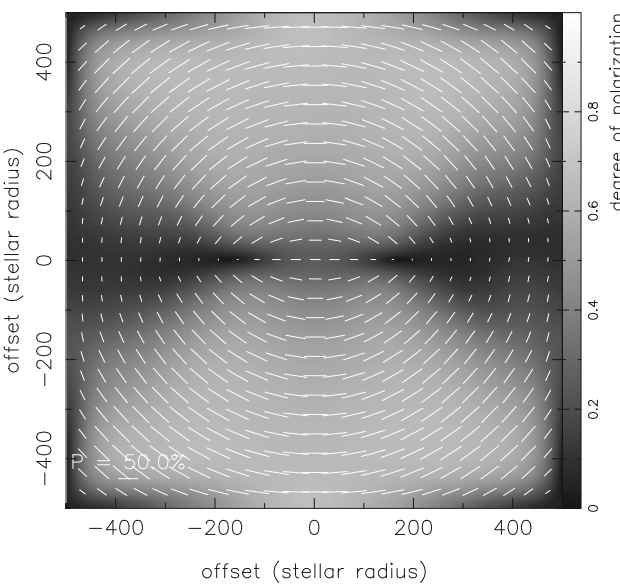

offset (stellar radius)
Fig. A.1. Test results of the total intensity images (upper panels) and polarization images (lower panels) at $\lambda=2.2 \mu \mathrm{m}$. The contour lines are overlaid in the intensity images with increments of 0.5 mag. The left and right columns show an optically thin case $(\tau=1)$ and an optically thick case $(\tau=10)$, respectively. Each image is convolved with a Gaussian function with a $F W H M$ of $200 R_{\star}$. of polarization. Polarization null points and polarization vector alignment are caused by the PSF smoothing effect in this model (see also the paper by Gledhill 2005). In fact, these signatures are not seen in the raw image and become more notable when the PSF size gets larger.

\section{References}

Bastien, P., \& Ménard, F. 1988, ApJ, 326, 334

Bertie, J. E., Labbé, H. J., \& Whalley, E. 1969, J. Chem. Phys., 50, 4501

Beuzit, J.-L., Thébault, P., Perrin, G., \& Rouan, D. 1994, A\&A, 291, L1

Bohren, C. F., \& Huffmann, D. R., 1983, Absorption and scattering of light by small particles (New York: John Wiley \& Sons)

Bujarrabal, V., Neri, R., Alcolea, J. \& Kahane, C. 2003, A\&A, 409, 573

Castro-Carrizo, A., Bujarrabal, V., Sánchez Contreras, C., Sahai, R., \& Alcolea, J. 2005, A\&A, 431, 979

De Ruyter, S., Van Winckel, H., Maas, T., et al. 2006, A\&A, 448, 641

Davis, C., Smith, M. D., Gledhill, T. M., \& Varricatt, W. P. 2005, MNRAS, 360, 104

Dominik, C., Gail, H.-P., \& Sedlmayr, E. 1989, A\&A, 223, 227

Dougados, C., Rouan, D., Lacombe, F., Forveille, T., \& Tiphene, D. 1990, A\&A, 227,437

Draine, B. T. 1985, ApJS, 57, 587

Fischer, O. Henning, Th., \& Yorke, H. W. 1994, A\&A, 284, 187

Forveille, T., Morris, M., Omont, A., \& Likkel, L. 1987, A\&A, 176, L13

Geballe, T. R., Kim, Y. H., Knacke, R. F., \& Noll, K. S. 1988, ApJ, 326, L65

Gledhill, T. M. 2005, MNRAS, 356, 883

Hodapp, K.-W., Sellgren, K., \& Nagata, T. 1988, ApJ, 326, L61

Hofmann, K.-H., \& Weigelt, G. 1986, A\&A, 167, L15

Hough, J. H., Whittet, D. C. B., Sato, S., et al. 1989, MNRAS, 241, 71

Jura, M. 1996, ApJ, 472, 806

Jura, M., Turner, J., \& Balm, S. P. 1997, ApJ, 474, 741

Jura, M., Turner, J. L., Van Dyk, S., \& Knapp, G. R. 2000a, ApJ, 528, L105

Jura, M., Chen, C., \& Werner, M. W. 2000b, ApJ, 541, 264

Kahn, F. D., \& West, K. A. 1985, MNRAS, 212, 837

Knapp, G. R. 1985, ApJ, 293, 273

Kozasa, T., Blum, J., \& Mukai, T. 1992, A\&A, 263, 423

Kozasa, T., Blum, J., Okamoto, H., \& Mukai, T. 1993, A\&A, 276, 278

Kwok, S. 1993, ARA\&A, 31, 63
Labeyrie, A. 1970, A\&A, 6, 85

Langill, P. P., Kwok, S., \& Hrivnak, B. J. 1994, PASP, 106, 736

Lohmann, A, W., Weigelt, G., \& Wirnitzer, B. 1983, Appl. Opt., 22, 4028

Lopez, B., \& Perrin, J.-M. 2000, A\&A, 354, 657

Lopez, B., Tuthill, P. G., Danchi, W. C., Monnier, J. D., \& Niccolini, G. 2001, A\&A, 377, 90

Mathis, J. S., Rumpl, W., \& Nordsieck, K. H. 1977, ApJ, 217, 425

Mauron, N., Le Borgne, J.-F., \& Picquette, M. 1989, A\&A, 218, 213

Meixner, M., Ueta, T., Dayal, A., et al. 1999, ApJ, 122, 221

Men'shchikov, A. B., Schertl, D., Tuthill, P. G., Weigelt, G., \& Yungelson, L. R. 2002, A\&A, 393, 867

Min, M., Dominik, C., Hovenier, J. W., de Koter, A., \& Waters, L. B. F. M. 2006, A\&A, 445, 1005

Morris, M., \& Reipurth, B. 1990, PASP, 102, 446

Murakawa, K., Suto, H., Tamura, M., et al. 2004, PASJ, 56, 509

Murakawa, K., Suto, H., Oya, S., et al. 2005, A\&A, 436, 601

Murakawa, K., Nakashima, J., Ohnaka, K., \& Deguchi, S. 2007, A\&A, 470, 957

Ohnaka, K., Driebe, T., Hofmann, K.-H., et al. 2006, A\&A, 445, 1015

Omont, A., Moseley, S. H., Forveille, T., et al. 1990, ApJ, 355, L27

Piirola, V., Scaltriti, F., \& Coyne, G. V. 1992, Nature, 359, 339

Robinson, G., Smith, R. G., \& Hyland, A. R. 1992, MNRAS, 256, 437

Roddier, F., Roddier, C., Graves, J. E., \& Northcott, M. J. 1995, ApJ, 443, 249

Rouan, D., Omont, A., Lacombe, F., \& Forveille, T. 1988, A\&A, 189, L3

Sahai, R., Bujarrabal, V., Castro-Carrizo, A., \& Zijlstra, A. 2000, A\&A, 360, L9

Sánchez Contreas, C., Le Mignant, D., Sahai, R., Gil de Paz, A., \& Morris, M. 2007, ApJ, 656, 1150

Scarrott, S. M., \& Scarrott, R. M. J. 1994, MNRAS, 268, 615

Smith, R. G., Sellgren, K., \& Tokunaga, A. T. 1988, ApJ, 334, 209

Unavane, M., Gilmore, G., Epchtein, N., et al. 1998, MNRAS, 295, 119

Ueta, T., \& Meixner, M. 2003, ApJ, 586, 1338

Voshchinnikov, N. V., Il'in, V. B., \& Henning, T. 2005, A\&A, 429, 371

Voshchinnikov, N. V., \& Henning, T. 2008, A\&A, 483, L9

Weigelt, G. 1977, Opt. Comm., 21, 55

Whittet, D. C. B., Martin, P. G., Hough, J. H., et al. 1992, ApJ, 386, 562

Whitney, B. A., \& Wolff, M. J. 2002, ApJ, 574, 205

Witt, A. N. 1977, ApJS, 35, 1

Wright, E. L. 1987, ApJ, 320, 818

Yamamura, I., Dominik, C., de Jong, T., Waters, L. B. F. M., \& Molster, F. J. 2000, A\&A, 363, 629 